Faculty of Applied Mathematics

University of Twente

University for Technical and Social Sciences
P.O. Box 217 7500 AE Enschede

The Netherlands

Phone:

+31-53-4893400

Fax: +31-53-4893114

Email:

memo@math.utwente.nl

Memorandum No. 1372

Various results on the toughness of graphs

H.J. Broersma, E.A. Engbers and H. Trommel

FEBRUARY 1997

ISSN 0169-2690 


\title{
Various results on the toughness of graphs
}

\author{
HAJO BRoERSMA * \\ ERIK ENGBERS ${ }^{\dagger}$ \\ Huib Trommel \\ Faculty of Applied Mathematics \\ University of Twente \\ P.O. Box 217, 7500 AE Enschede, The Netherlands
}

January 15, 1998

\begin{abstract}
Let $G$ be a graph, and let $t \geq 0$ be a real number. Then $G$ is $t$-tough if $t \omega(G-S) \leq|S|$ for all $S \subseteq V(G)$ with $\omega(G-S)>1$, where $\omega(G-S)$ denotes the number of components of $G-S$. The toughness of $G$, denoted by $\tau(G)$, is the maximum value of $t$ for which $G$ is $t$-tough (taking $\tau\left(K_{n}\right)=\infty$ for all $n \geq 1$ ). $G$ is minimally $t$-tough if $\tau(G)=t$ and $\tau(H)<t$ for every proper spanning subgraph $H$ of $G$. We discuss how the toughness of (spanning) subgraphs of $G$ and related graphs depends on $\tau(G)$, we give some sufficient (degree) conditions implying $\tau(G) \geq t$, and we study which subdivisions of 2-connected graphs have minimally 2 -tough squares.
\end{abstract}

Keywords: $t$-tough graph, toughness (of subgraphs), minimally $t$-tough graph, square (of a) graph, degree sum condition.

AMS Subject Classifications (1991): 05C75, 05C35.

\section{Introduction}

In 1973, Chvátal [5] introduced a new graph invariant called toughness, and he investigated its relation to the existence of Hamilton cycles. Although many results concerning the relation between toughness and cycle structure have been obtained since, the main conjectures raised in [5] are still open, and little progress has been made towards (dis)proving these conjectures.

${ }^{*}$ Corresponding author. E-mail: broersma@math.utwente.nl

${ }^{\dagger}$ Present address: Faculty WINS, University of Amsterdam, Kruislaan 403, 1098 SJ Amsterdam, NL. 
More recently, toughness has also been considered as a vulnerability measure for networks, since it measures how badly a graph breaks up in components when a set of vertices is removed. Unfortunately, this measure is difficult to calculate: as shown by Bauer et al. [2] it is NP-hard to determine the toughness of a graph.

Despite these negative observations, many new results involving the toughness of a graph have appeared, and new and interesting ideas have been added to the field, in an attempt to solve the main problems and to get more grip on the concept of toughness. The results presented here should be considered in the same vein.

\section{Preliminaries}

We start this section with some terminology and notation. We refer to [3] and [9] for any undefined terminology and notation, and consider finite undirected graphs without loops and multiple edges only.

Let $G$ be a graph, and let $t \geq 0$ be a real number. Then $G$ is $t$-tough if $t \omega(G-S) \leq|S|$ for all $S \subseteq V(G)$ with $\omega(G-S)>1$, where $\omega(G-S)$ denotes the number of components of $G-S$. The toughness of $G$, denoted by $\tau(G)$, is the maximum value of $t$ for which $G$ is $t$-tough (taking $\tau\left(K_{n}\right)=\infty$ for all $\left.n \geq 1\right)$. A set $S \subseteq V(G)$ with the property that $\tau(G)=\frac{|S|}{\omega(G-S)}$ is called a toughness determining set of $G$. A Hamilton cycle (Hamilton path) of $G$ is a cycle (path) of $G$ containing every vertex of $G ; G$ is called hamiltonian if it contains a Hamilton cycle, and hamiltonian-connected if between any two vertices of $G$ there exists a Hamilton path of $G$.

The concept of toughness was introduced in [5], where relations between toughness and the existence of Hamilton cycles or $k$-factors ( $k$-regular spanning subgraphs) are studied, and several conjectures are stated. The following conjecture is still open.

\section{Conjecture 1}

Every 2-tough graph on at least 3 vertices is hamiltonian.

Little progress has been made towards a proof of Conjecture 1, although in [7] it is shown that every 2 -tough graph (on at least 3 vertices) contains a 2 -factor, and that there exist $(2-\epsilon)$-tough graphs not containing a 2-factor (hence non-hamiltonian) for arbitrarily small $\epsilon>0$.

In [1] it is shown that Conjecture 1 is equivalent to other statements about 2-tough graphs, including the next conjecture.

\section{Conjecture 2}

Every 2-tough graph is hamiltonian-connected.

The results in Section 5 are motivated by an attempt to find a counterexample to Conjecture 2 within the class of (spanning subgraphs of) squares of 2-connected graphs. The results in 
the other sections are based on an attempt to get more grip on the concept of toughness. In Section 3 we express the toughness of (spanning) subgraphs of a graph $G$ and related graphs in terms of the toughness of $G$. In the main result of Section 4 a certain value of the toughness is guaranteed by a degree sum condition.

\section{Toughness of subgraphs and related graphs}

\subsection{Toughness of spanning subgraphs}

In this section we will prove that every non-complete graph with toughness greater than one contains a spanning subgraph with toughness exactly one. In fact, we will prove a stronger result from which the above follows as a special case. One could wonder (as we did) whether for all non-complete graphs $G$ with $\tau(G)$ not an integer, there exists a spanning subgraph $H$ with $\tau(H)=\lfloor\tau(G)\rfloor$. We were not able to (dis)prove this.

In the next proof we use the following easy obervation. Let $S$ be a cut set of a graph $G$. Then $|S| \geq 2 \tau(G)[5]$.

\section{Theorem 3}

Let $G$ be a graph $\left(\neq K_{1}, K_{2}, \ldots, K_{\left\lfloor\frac{4 i+7}{3}\right\rfloor}\right)$ with $\tau(G)>i$ for some positive integer $i$. Then there exists a spanning subgraph $H$ of $G$ with $\frac{2 i+1}{3} \leq \tau(H) \leq i$.

Proof Suppose there does not exist a spanning subgraph $H$ of $G$ with $\frac{2 i+1}{3} \leq \tau(H) \leq i$. Let $H_{1}$ and $H_{2}$ be spanning subgraphs of $G$ such that $H_{2}=H_{1}-e$ for some $e \in E\left(H_{1}\right)$, and such that $\tau\left(H_{1}\right)>i$ and $\tau\left(H_{2}\right)<\frac{2 i+1}{3}$. Then there exists a cut set $S$ of $H_{2}$ for which $\tau\left(H_{2}\right)=\frac{|S|}{\omega\left(H_{2}-S\right)}<\frac{2 i+1}{3}$, and thus $\omega\left(H_{2}-S\right)>\frac{3|S|}{2 i+1}$. Since $\omega\left(H_{2}-S\right)$ and $3|S|$ are integers, we get $\omega\left(H_{2}-S\right) \geq \frac{3|S|+1}{2 i+1}$. First suppose $S$ is a cut set of $H_{1}$. Then $\omega\left(H_{1}-S\right) \geq \omega\left(H_{2}-S\right)-1 \geq$ $\frac{3|S|+1}{2 i+1}-1$. On the other hand, since $\tau\left(H_{1}\right)>i, \omega\left(H_{1}-S\right)<\frac{|S|}{i}$, hence $\omega\left(H_{1}-S\right) \leq \frac{|S|-1}{i}$. Thus we get $\frac{3|S|+1-2 i-1}{2 i+1} \leq \frac{|S|-1}{i}$. For $i=1$ this clearly leads to a contradiction. So let $i>1$. Then, equivalently, $3 i|S|-2 i^{2} \leq 2 i|S|+|S|-2 i-1$, hence $(i-1)|S| \leq 2 i^{2}-2 i-1$. So the following holds for $|S|:|S| \leq \frac{2 i^{2}-2 i-1}{i-1}=2 i-\frac{1}{i-1}<2 i$. But, on the other hand, $|S| \geq 2 \tau\left(H_{1}\right)>2 i$, a contradiction. This leaves us with the case that $S$ is not a cut set of $H_{1}$. This means $e$ (in $H_{1}$ ) connects the only two components of $H_{2}-S$. Since $S$ is a toughness determining set of $H_{2}, \omega\left(H_{2}-S\right)=2$, and since $\tau\left(H_{2}\right)<\frac{2 i+1}{3},|S|<\frac{4 i+2}{3}$, hence $|S| \leq \frac{4 i+1}{3}$. If $S \cup\{u\}$ is a cut set of $H_{1}$ for some $u \in V\left(H_{1}\right)$, then $|S \cup\{u\}| \geq 2 \tau\left(H_{1}\right)>2 i$, so $|S| \geq 2 i$. This contradicts $|S| \leq \frac{4 i+1}{3}$ since $i \geq 1$. This implies that the two components of $H_{2}-S$ are $K_{1}$. But then $|V(G)|=|S|+2 \leq \frac{4 i+1}{3}+2=\frac{4 i+7}{3}$, thus $|V(G)| \leq\left\lfloor\frac{4 i+7}{3}\right\rfloor$. This implies that $G$ is not complete. But then $\tau(G) \leq \frac{|V(G)|-2}{2} \leq \frac{4 i+1}{6}<i$, our final contradiction.

The lower bound on $\tau(H)$ in Theorem 3 cannot be raised. Let $G$ be a $K_{\frac{4 i+8}{3}}$. Then for every spanning subgraph $H$ of $G, \tau(H) \leq \tau(G-e)=\frac{\frac{4 i+8}{3}-2}{2}=\frac{2 i+1}{3}$. We do not know whether 
there exist non-complete examples showing this. In fact, it could be true that for every noncomplete graph $G$ with $\tau(G)$ not an integer, there exists a spanning subgraph $H$ of $G$ with $\tau(H)=\lfloor\tau(G)\rfloor$.

By taking $i=1$ in Theorem 3, we get the following result as a special case.

\section{Corollary 4}

Let $G$ be a graph $\left(\neq K_{1}, K_{2}, K_{3}\right)$ with $\tau(G)>1$. Then there exists a spanning subgraph $H$ of $G$ with $\tau(H)=1$.

\subsection{Toughness of subgraph related graphs}

The next two lemmas give a relation between the toughness of a graph $G$ and the toughness of the join of a (small) complete graph and a component of $G-S$, where $S$ is a toughness determining set of $G$. These lemmas might turn out to be useful in inductive proofs of structural results on graphs with a certain toughness.

By $G \vee H$ we denote the join of two disjoint graphs $G$ and $H$, i.e. the graph obtained from $G$ and $H$ by joining every vertex of $G$ to every vertex of $H$.

First we give a result for arbitrary toughness determining sets.

\section{Lemma 5}

Let $k$ be a positive integer, and let $G$ be a graph with $\tau(G) \geq k, S$ an arbitrary toughness determining set of $G$, and $H_{i}$ an arbitrary component of $G-S$. Then $\tau\left(K_{k} \vee H_{i}\right) \geq k$.

Proof Suppose $\tau\left(K_{k} \vee H_{i}\right)<k$. Then there exists a subset $S_{i}^{\prime} \subset V\left(K_{k} \vee H_{i}\right)$ such that $\left|S_{i}^{\prime}\right|<k \omega\left(\left(K_{k} \vee H_{i}\right)-S_{i}^{\prime}\right)$. Denote the vertices of $K_{k} \vee H_{i}$ which are not a vertex of $H_{i}$ by $v_{1}, \ldots, v_{k}$. Define $S_{i}=S_{i}^{\prime} \backslash\left\{v_{1}, \ldots, v_{k}\right\}$. Then $\left|S_{i}\right|=\left|S_{i}^{\prime}\right|-k$ and $\omega\left(\left(K_{k} \vee H_{i}\right)-S_{i}^{\prime}\right)=\omega\left(H_{i}-S_{i}\right)$.

Now

$$
\tau(G) \leq \frac{\left|S \cup S_{i}\right|}{\omega\left(G-\left(S \cup S_{i}\right)\right)}=\frac{|S|+\left|S_{i}\right|}{\omega(G-S)+\omega\left(H_{i}-S_{i}\right)-1}<\frac{|S|+\left|S_{i}\right|}{\omega(G-S)+\frac{\left|S_{i}\right|}{k}} \leq \frac{|S|}{\omega(G-S)},
$$

a contradiction.

To show that the lower bound on $\tau\left(K_{k} \vee H_{i}\right)$ in Lemma 5 cannot be increased, consider e.g. the graph $G_{p, k}(p \geq 2, k \geq 1)$ obtained by joining a $K_{p k}$ to $p$ disjoint copies of a $K_{k+2}$ with one edge $e$ deleted. Then $\tau\left(G_{p, k}\right)=\frac{p k}{p}=\frac{(p+1) k}{p+1}=\ldots=\frac{2 p k}{2 p}=k$. Let $S=V\left(K_{p k}\right)$. Then $H_{i}=K_{k+2}-e$ and $\tau\left(K_{k} \vee H_{i}\right)=\tau\left(K_{k} \vee\left(K_{k+2}-e\right)\right)=\frac{k+k}{2}=k$.

Although Lemma 5 is sharp, it can be improved in the following sense if we only look at maximum toughness determining sets. Here it is convenient to adopt the notation that $K_{k-1} \vee H_{i}=H_{i}$ if $k=1$.

\section{Lemma 6}

Let $k$ be a positive integer, and let $G$ be a graph with $\tau(G) \geq k, S$ a maximum toughness determining set of $G$, and $H_{i}$ an arbitrary component of $G-S$. Then $\tau\left(K_{k-1} \vee H_{i}\right) \geq k$. 
Proof Suppose $\tau\left(K_{k-1} \vee H_{i}\right)<k$. Then, as in the proof of the previous lemma, there now exists a nonempty set $S_{i} \subset V\left(H_{i}\right)$ such that $\frac{\left|S_{i}\right|-1}{k}+1<\omega\left(H_{i}-S_{i}\right)$. Hence $\omega\left(H_{i}-S_{i}\right) \geq 1+\frac{\left|S_{i}\right|}{k}$. So

$$
\tau(G)<\frac{\left|S \cup S_{i}\right|}{\omega\left(G-\left(S \cup S_{i}\right)\right)}=\frac{|S|+\left|S_{i}\right|}{\omega(G-S)+\omega\left(H_{i}-S_{i}\right)-1} \leq \frac{|S|+\left|S_{i}\right|}{\omega(G-S)+\frac{\left|S_{i}\right|}{k}} \leq \frac{|S|}{\omega(G-S)},
$$

a contradiction.

To show that the lower bound on $\tau\left(K_{k-1} \vee H_{i}\right)$ in Lemma 6 cannot be increased, consider e.g. the graph $H_{p, k}(p \geq 2, k \geq 1)$ obtained by joining a $K_{p k}$ to $p$ disjoint copies of a $C_{4}$ joined to a $K_{k-1}$ if $k \geq 2$. Then $\tau\left(H_{p, k}\right)=k$. Let $S=V\left(K_{p k}\right)$. Then $S$ is a maximum toughness determining set and $H_{i}=K_{k-1} \vee C_{4} ; \tau\left(K_{k-1} \vee H_{i}\right)=k$.

\section{Sufficient conditions for $t$-toughness}

In this section we present some sufficient conditions guaranteeing a graph to be $t$-tough.

Chvátal and Erdös [6] proved that a graph $G$ on at least three vertices with $\alpha(G) \leq \kappa(G)$ is hamiltonian, hence 1-tough. It is very easy to extend this result to $t$-toughness.

\section{Lemma 7}

Let $G$ be a graph. If $t \alpha(G) \leq \kappa(G)$, then $G$ is $t$-tough.

Proof Suppose $G$ is not $t$-tough. Then there exists a cut set $S$ of $G$ with $\omega(G-S)>\frac{|S|}{t}$. Clearly $\alpha(G) \geq \omega(G-S)$, so $\alpha(G)>\frac{|S|}{t}$, which implies $t \alpha(G)>|S| \geq \kappa(G)$.

The connectivity condition in Lemma 7 cannot be relaxed (and still guarantee $t$-toughness), as can easily be seen from the graph $K_{p} \vee \bar{K}_{q}$ with $p>q$ : $\alpha=q, \kappa=p$ and $\tau=\frac{p}{q}=\frac{\kappa}{\alpha}$.

In a similar way, one might try to extend degree conditions for hamiltonicity (hence 1toughness) to results for $t$-toughness, e.g. the following result of Ore [11].

\section{Theorem 8}

Let $G$ be a graph on $n \geq 3$ vertices such that for every two nonadjacent vertices $u$ and $v$ of $G$, $d(u)+d(v) \geq n$. Then $G$ is hamiltonian.

The degree condition in this theorem can be changed as follows to obtain a degree condition which implies $t$-toughness. In the following result we use $\sigma_{k}(G)$ to denote the minimum degree sum of the vertices of an independent set with $k$ vertices of the graph $G$ (if such exists; otherwise we define $\left.\sigma_{k}=k(|V(G)|-1)\right)$.

\section{Theorem 9}

Let $G$ be a $k t$-connected graph ( $k$ and $k t$ integers and $k t \geq 1$ ) on $n$ vertices with $\sigma_{k+1} \geq$ $(k+1)\left(\frac{n t}{t+1}\right)$. Then $G$ is $t$-tough. 
Proof Suppose $G \neq K_{n}$ and $G$ is not $t$-tough. Then for some $S \subset V(G): \omega(G-S)>\frac{|S|}{t}$. Suppose $\omega(G-S)<k+1$, then $\frac{|S|}{t}<\omega(G-S) \leq k$, implying $\kappa(G) \leq|S|<k t$, a contradiction. So $\omega(G-S) \geq k+1$. Let $v_{1}, v_{2}, \ldots, v_{k+1}$ denote $k+1$ vertices from distinct components of $G-S$. Then $(k+1)\left(\frac{n t}{t+1}\right) \leq \sigma_{k+1} \leq \sum_{i=1}^{k+1} d\left(v_{i}\right) \leq(k+1)|S|+n-|S|-\omega(G-S)<k|S|+n-\frac{|S|}{t}$. Hence $(k+1) t n<k(t+1)|S|+n(t+1)-\frac{|S|(t+1)}{t}$, and $(k t-1) n<\frac{k t(t+1)}{t}|S|-\left(\frac{t+1}{t}\right)|S|=$ $(k t-1) \frac{t+1}{t}|S|$. If $k t=1$, we obtain a contradiction. Hence assume $k t>1$. Then $|S|>\frac{t n}{t+1}$, hence $\omega(G-S)>\frac{|S|}{t}>\frac{n}{t+1}$. But now $n \geq|S|+\omega(G-S)>\frac{t n}{t+1}+\frac{n}{t+1}=n$, a contradiction.

Note that if $G$ is $k t$-connected with $k \geq \alpha(G)$, then we know that $G$ is $t$-tough (by Lemma 7 ). In case $k<\alpha(G), G$ has an independent set with $k+1$ vertices, and the degree sum condition makes sense.

From Theorem 9 we can simply derive an extension of Theorem 8 to $t$-toughness. To show that it is an extension, we need the following lemma.

\section{Lemma 10}

Let $G$ be a graph on $n$ vertices such that for every two nonadjacent vertices $u$ and $v$ of $G$, $d(u)+d(v) \geq \frac{2 n t}{t+1}$ with $1 \leq t \leq n-1$. Then $G$ is $\lceil t\rceil$-connected.

Proof Suppose $G$ is not $\lceil t\rceil$-connected. Then there exists a vertex cut $S \subset V(G)$ with $|S|<\lceil t\rceil$. Since $|S|$ is an integer, this implies $|S| \leq\lceil t\rceil-1<t$. Since $S$ is a vertex cut, $G-S$ consists of at least two components. Choose a vertex $u$ in one component and a vertex $v$ in another component. Then $d(u)+d(v) \leq 2|S|+n-2-|S|=n+|S|-2<n+t-2$, so $d(u)+d(v)<n+t-2$. Substituting the degree condition for $u$ and $v$, we obtain $\frac{2 n t}{t+1}<n+t-2$, or, equivalently, $(n-t)(t-1)<-2$, which is absurd since $1 \leq t \leq n-1$.

\section{Corollary 11}

Let $G$ be a graph on $n$ vertices such that for every two nonadjacent vertices $u$ and $v$ of $G$, $d(u)+d(v) \geq \frac{2 n t}{t+1}$ with $1 \leq t \leq n-1$. Then $G$ is $t$-tough.

Corollary 11 generalizes a result of Bauer et al. [2] in which the minimum degree condition $\delta(G) \geq \frac{n t}{t+1}$ is shown to be sufficient for $t$-toughness.

\section{Spanning subgraphs of squares}

One possible approach to disproving the 2-tough-conjecture (Conjecture 1) would be to try to disprove the equivalent conjecture that all 2-tough graphs are hamiltonian-connected (Conjecture 2), i.e. by finding a graph which is 2-tough but not hamiltonian-connected. To avoid the difficulty of checking whether the considered graphs are 2-tough (which is an NP-hard problem $[2]$ ), one could restrict the search for a counterexample to a class of graphs every member of 
which is known to be 2-tough. An example of such a class of graphs is the class consisting of the squares of all 2-connected graphs. The square $G^{2}$ of a graph $G$ is the graph obtained from $G$ by joining all vertices at distance 2 in $G$. By an elementary result of Chvátal [5], the square of a $k$-connected graph is $k$-tough. Unfortunately, the square of a 2 -connected graph is also hamiltonian-connected, by an observation in [4] based on the beautiful deep result due to Fleischner [8] that all squares of 2-connected graphs are hamiltonian. So all attempts to find a counterexample within this class of squares of 2-connected graphs will fail, that is, if we restrict ourselves to graphs within this class. But, by the ease of showing that squares of 2-connected graphs are 2-tough, and the difficulty of showing that these squares are hamiltonian-connected, one could be tempted to think that there exist spanning subgraphs of these squares that are 2-tough, but not hamiltonian-connected. This led us to define and study minimally 2-tough graphs, or, more generally, minimally $t$-tough graphs. A graph $G$ is called minimally $t$-tough if $\tau(G)=t$ and there does not exist a proper spanning subgraph $H$ of $G$ with $\tau(H)=t$. By the above motivation, we concentrate on squares of 2-connected graphs and consider the problem which of these graphs are (not) minimally 2-tough. We need one more definition. Let $G$ and $H$ be graphs, and $s$ an integer with $s \geq 0$. $G$ is an $s$-subdivision of $H$ if $G$ can be obtained from $H$ by replacing every edge $u v$ of $H$ by a path between $u$ and $v$ with at least $s$ internal vertices. We call $G$ an $s$-subdivision if it is an $s$-subdivision of some graph $H$. Clearly, a graph which is an $s$-subdivision $(s \geq 1)$ is also an $(s-1)$-subdivision, and an $s$-subdivision of a 2-connected graph is 2-connected.

As mentioned by Van den Heuvel [10] during the Workshop on the Hamiltonicity of 2Tough Graphs held at Enschede, The Netherlands in November 1995, sponsored by EIDMA (the Euler Institute for Discrete Mathematics and its Applications), "sufficiently" subdivided 2-connected 3-regular graphs have minimally 2-tough squares. In this section we will further specify what "sufficiently" subdivided means.

To prove that the square of a 4-subdivision of a 2-connected 3-regular graph is minimally 2 -tough, we first prove that for the square $G$ of a 2-connected 3-subdivision $H, \tau(G)=2$.

\section{Lemma 12}

Let $H$ be a 3-subdivision of a 2-connected graph and let $G=H^{2}$. Then $\tau(G)=2$.

Proof From [5] we know that the square of every 2-connected graph is 2-tough, which means $\tau(G) \geq 2$. Suppose $H=C_{n}$. Then every vertex in $G$ has degree 4 , thus $\tau(G) \leq 2$. So suppose $H \neq C_{n}$. Now label the vertices of $G$ with degree exceeding 2 in $H$ as $v_{1}, v_{2}, \ldots, v_{k}$. Construct $S$ such that it contains all the vertices $v_{i}$ and, for every $v_{i}, d_{H}\left(v_{i}\right)-1$ vertices which are neighbors of $v_{i}$ in $H$. Here $d_{H}\left(v_{i}\right)$ denotes the degree of $v_{i}$ in $H$. Then $|S|=\sum_{i=1}^{k} d_{H}\left(v_{i}\right)$. By deleting $S$ from $G$, there will be a component between any two vertices $v_{i}, v_{j}$ of $G$ which are neighbors in $H$. So $\omega(G-S)=\frac{1}{2} \sum_{i=1}^{k} d_{H}\left(v_{i}\right)$, and $\tau(G) \leq \frac{|S|}{\omega(G-S)}=2$. 


\section{Theorem 13}

Let $H$ be a 4-subdivision of a 2-connected 3-regular graph and let $G=H^{2}$. Then $G$ is minimally 2-tough.

Proof From Lemma 12 we know that $\tau(G)=2$, so it is sufficient to prove that $\tau(G-e)<2$ for an arbitrary edge $e \in E(G)$. This is clear if $e$ is incident with a vertex of degree 4 in $G$. Construct $S$ as is Lemma 12, and consider the next three remaining possibilities for $e$. First assume $e$ is incident with two vertices $w_{1} \in S$ and $w_{2} \in S$ of degree 5 . Denote the common neighbor of $w_{1}$ and $w_{2}$ with degree 5 by $w_{3}$, and define $S^{\prime}=\left(S \cup\left\{w_{3}\right\}\right) \backslash\left\{w_{1}, w_{2}\right\}$. Then $\left|S^{\prime}\right|=|S|-1$ and $\omega\left((G-e)-S^{\prime}\right)=\omega(G-S)$. So $\tau(G-e) \leq \frac{\left|S^{\prime}\right|}{\omega\left((G-e)-S^{\prime}\right)}<\frac{|S|}{\omega(G-S)}=2$. Next assume $e$ is incident with two vertices $w_{1} \in S$ and $w_{2} \notin S$ of degree 5 . Define $S^{\prime}=S \backslash\left\{w_{1}\right\}$. As in the previous case, $\tau(G-e)<2$. Finally assume $e$ is incident with a vertex $w_{1}$ of degree 6 and a vertex $w_{2}$ of degree 5 . Denote by $P$ the path in $H$ containing $e$ between $w_{1}$ and the next vertex $x$ of degree 3 in $H$. Note that we may choose $S$ in such a way that the predecessor of $x$ on $P$ is not in $S$. Define $S^{\prime}=\left(S \cup N_{G}\left(w_{1}\right) \cup N_{G}\left(w_{2}\right)\right) \backslash\left\{w_{1}, w_{2}\right\}$. Then $\tau(G-e) \leq \frac{\left|S^{\prime}\right|}{\omega\left((G-e)-S^{\prime}\right)}=\frac{|S|+3}{\omega(G-S)+2}<2$.

We will now prove that if two neighbors $u$ and $v$ with $d(u)+d(v) \geq 6$ exist in a 2-connected graph $H$, then $H^{2}$ is not minimally 2-tough. To prove that, we need the following lemma. A graph $G$ is minimally 2-connected if $\kappa(G)=2$ and $\kappa(H)<2$ for every proper spanning subgraph of $G$.

\section{Lemma 14}

Let $G=H^{2}$ be a minimally 2-tough graph. Then $H$ is minimally 2-connected and triangle-free.

Proof Suppose $H$ is not minimally 2-connected. Then there exists a proper spanning subgraph $F$ of $H$ which is 2-connected. But then $F^{2}$ is a proper spanning subgraph of $G$ and $F^{2}$ is 2-tough, a contradiction. Next suppose $H$ has a triangle uvwu. Because $H$ is minimally 2-connected, $w$ is a cut vertex of $H-u v$. Denote the components of $(H-u v)-w$ as $H_{1}$ (the component which contains $u$ ) and $H_{2}$ (the component which contains $v$ ). Notice that there are no other components of $(H-u v)-w$, otherwise $H-w$ would be disconnected. By similar arguments, $v$ is a cut vertex of $H-u w$ but not of $H$. Because $H_{1}$ is connected, there are no edges between $w$ and $H_{1}$ other than $u w$, otherwise $H-u w$ would not have $v$ as a cut vertex. By symmetry, there are no edges between $H_{2}$ and $w$ other than $v w$. Now $H_{1}$ can only contain $u$ and $H_{2}$ can only contain $v$, otherwise $u$ or $v$ would be a cut vertex of $H$. So $H=K_{3}$, a contradiction, since then $\tau(G) \neq 2$.

\section{Theorem 15}

Let $H$ be a 2-connected graph with $d_{H}(u)+d_{H}(v) \geq 6$ for some $u, v \in V(H)$ with $u v \in E(H)$, and let $G=H^{2}$. Then $G$ is not minimally 2-tough. 
Proof Suppose $G$ is minimally 2-tough. Then $\tau(G-e)<2$ for every $e \in E(G)$, so also for $e=u v$. Hence there exists a vertex cut $S \subset V(G-e)$ such that $\omega((G-e)-S)>\frac{|S|}{2}$. Since $\omega$ is an integer, $\omega((G-e)-S) \geq\left\lceil\frac{|S|+1}{2}\right\rceil=\left\lfloor\frac{|S|}{2}\right\rfloor+1$. First suppose $S$ is a vertex cut of $G$. Since $G$ is 2-tough, we have $\omega(G-S) \leq\left\lfloor\frac{|S|}{2}\right\rfloor$, which implies $\omega((G-e)-S)=\left\lfloor\frac{|S|}{2}\right\rfloor+1$, and $\omega(G-S)=\left\lfloor\frac{\lfloor S \mid}{2}\right\rfloor$. Two vertices of two distinct components of $G-S$ have no common neighbors in $S$ in $H$, otherwise in $G$ there would be an edge between these components. Since $H$ is $2-$ connected, every component other than the component containing $e$, has at least two distinct neighbors in $S$ in $H$. Now consider the number of neighbors in $S$ in $H$ of the component of $G-S$ containing $e$. Since $d_{H}(u)+d_{H}(v) \geq 6$ and $u$ and $v$ cannot have neighbors in $H$ in the components of $(G-e)-S$, except by the edge e, $\left|N_{H}(u) \cap S\right|+\left|N_{H}(v) \cap S\right| \geq 4$. If $\left|N_{H}(u) \cap N_{H}(v) \cap S\right| \neq 0$, then $H$ contains a triangle. This is impossible by Lemma 14 . Hence the component of $G-S$ containing $e$ has at least four distinct neighbors in $S$ in $H$. We conclude that $|S| \geq 2\left(\left\lfloor\frac{|S|}{2}\right\rfloor-1\right)+4=2\left(\left\lfloor\frac{|S|}{2}\right\rfloor+1\right) \geq 2\left(\frac{|S|-1}{2}+1\right)=|S|+1$, a contradiction.

Next suppose $S$ is not a vertex cut of $G$. Then $2=\omega((G-e)-S)>\frac{|S|}{2}$, implying that $|S| \leq 3$. But for the same reasons as before, $u$ and $v$ have together at least four distinct neighbors in $S$ in $H$, a contradiction.

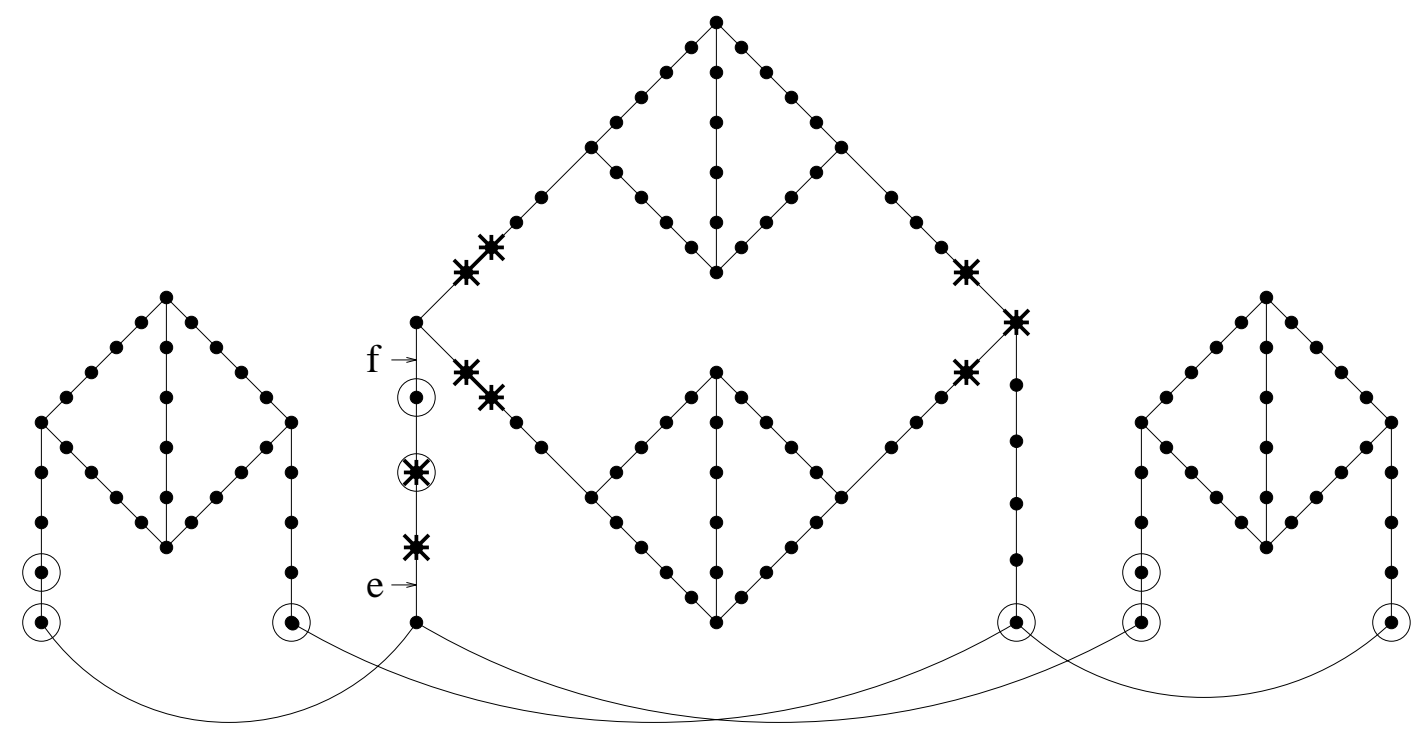

Figure 1: The square of this graph is minimally 2-tough.

So squares of 4-subdivisions of 3-regular 2-connected graphs are minimally 2-tough and squares of 2-connected graphs with at least one vertex of degree exceeding 3 are 2-tough, but not minimally 2-tough, and the same holds for squares of 2-connected graphs which have two adjacent vertices with degree sum 6 or more. What can we say about squares of $s$-subdivisions with $s \leq 3$ ? There are examples of squares of 3-subdivisions which are minimally 2-tough and examples of squares of 3 -subdivisions which are not minimally 2 -tough. If we take the square 
of the 3 -subdivision drawn in Figure 1, the resulting graph $G$ is minimally 2 -tough.

To prove this, it is sufficient to show that deletion of the marked edge $e$ or $f$ decreases the toughness. All other edges are incident with a vertex of degree 4 , or can be proved to decrease the toughness in the same way, or as in the proof of Theorem 13. $\tau(G-e) \leq \frac{9}{5}<2$ due to the vertex cut marked with circles in the figure and $\tau(G-f) \leq \frac{9}{5}<2$ due to the vertex cut marked with stars in the figure.

The example we give of a 3-subdivision whose square is not minimally 2-tough, is less spectacular. Let $H$ be the graph obtained from $K_{4}$ by replacing each edge by a path with exactly three internal vertices, and let $G=H^{2}$. Clearly, $G$ is 2-tough. But $G-e$ is also 2-tough, where $e$ is an edge of $H$ incident with a vertex of degree 3 in $H$ (we omit further details).

The above examples illustrate the difficulties of obtaining a full classification of all minimal 2-tough squares of 3 -subdivisions.

\section{References}

[1] D. Bauer, H.J. Broersma, J. van den Heuvel, H.J. Veldman, On hamiltonian properties of 2-tough graphs. J. Graph Theory 18 (1994) 539-543.

[2] D. Bauer, A. Morgana, E. Schmeichel, On the complexity of recognizing tough graphs. Discrete Math. 124 (1994) 13-17.

[3] J.A. Bondy, U.S.R. Murty, Graph Theory with Applications. (MacMillan/Elsevier, London/New York, 1976).

[4] G. Chartrand, A.M. Hobbs, H.A. Jung, The square of a block is Hamiltonian connected. J. Combinat. Theory B 16 (1974) 290-292.

[5] V. Chvátal, Tough graphs and hamiltonian circuits. Discrete Math. 5 (1973) 215-228.

[6] V. Chvátal, P. Erdös, A note on hamiltonian circuits. Discrete Math. 2 (1972) 111-113.

[7] H. Enomoto, B. Jackson, P. Katerinis, A. Saito, Toughness and the existence of $k$-factors. J. Graph Theory 9 (1985) 87-95.

[8] H. Fleischner, The square of every 2-connected graph is hamiltonian. J. Combinat. Theory B 16 (1974) 29-34.

[9] M.R. Garey, D.S. Johnson, Computers and Intractability, (W.H. Freeman and Company, New York, 1979).

[10] J. van den Heuvel, Personal communication (1995). Also in: EIDMA Workshop on Hamiltonicity of 2-Tough Graphs: Progress Report, J.A. Bondy, H.J. Broersma, C. Hoede, and H.J. Veldman (Editors). To appear in Networks.

[11] O. Ore, Note on hamiltonian circuits. Amer. Math. Monthly 67 (1960) 55. 